\title{
Los saberes de los ancestros: clave para los vínculos con la Madre Tierra en una comunidad andina en Argentina
}

\author{
Cornelia Steinhäuser \\ University of Münster. Institute of Landscape Ecology \\ cornelia.steinhaeuser@wwu.de
}

Recibido: mayo de 2019

Aceptado: noviembre de 2019

Publicado: marzo de 2020

\section{Resumen}

Si asumimos que los paisajes rurales agrobiodiversos se generan en permanente cocreación entre humanos y no humanos, es relevante reconocer las formas del saber inmersas en este proceso para diseñar la transformación socioecológica hacia un desarrollo sostenible, especialmente de cara a la transición a la agroecología. Este trabajo, un estudio de caso realizado en el norte argentino, aborda las relaciones tangibles e intangibles con las que una comunidad andina construye su realidad y un paisaje complejo y variado. El estudio utiliza metodologías etnográficas combinando la observación participante con entrevistas y el análisis cualitativo. El resultado hace visible los vínculos entre humanos y no humanos, narrados por las personas entrevistadas, como el afecto y la reciprocidad, que son decisivos para permanecer cultivando la tierra y desarrollarse endógenamente con sus saberes campesinos basándose en los ancestros. A su vez, se discute la compatibilidad de tales saberes - en cuyo origen se encuentra una plena inmersión en el paisaje- con los estilos de vida actuales.

Palabras clave: agroecología; formas del saber; paisajes rurales; relación entre humanos y naturaleza; transformación

Resum. Els sabers dels ancestres: clau per als vincles amb la Mare Terra en una comunitat andina a l'Argentina

Si assumim que els paisatges rurals agrobiodiversos es generen en permanent cocreació entre humans i no humans, és rellevant reconèixer les formes del saber immerses en aquest procés per dissenyar la transformació socioecològica cap a un desenvolupament sostenible, 
especialment de cara a la transició a l'agroecologia. Aquest treball, plantejat sobre la base d'un estudi de cas realitzat al nord argentí, aborda les relacions tangibles i intangibles amb les quals una comunitat andina construeix la seva realitat i un paisatge complex i variat. L'estudi utilitza metodologies etnogràfiques tot combinant l'observació participant amb entrevistes i l'anàlisi qualitativa. El resultat fa visible l'entramat de vincles entre humans i no humans narrat per les persones entrevistades, com ara l'afecte i la reciprocitat, que són decisius per romandre conreant la terra i desenvolupar-s'hi endògenament amb els seus sabers pagesos basats en els ancestres. Al seu torn, es discuteix la compatibilitat d'aquests sabers — a l'origen dels quals es troba una plena immersió en el paisatge- amb els estils de vida actuals.

Paraules clau: agroecologia; formes del saber; paisatges rurals; relació entre humans i naturalesa; transformació

Résumé. Les savoirs des ancêtres: Clé des liens avec la 'Terre Mère' dans une communauté andine en Argentine

Si nous supposons que les paysages de l'agrobiodiversité rurale sont générés par une co-création permanente entre humains et non-humains, il est pertinent de reconnaitre les formes de connaissance concernées par ce processus et ce essentiellement, pour concevoir la transformation socio-écologique vers un développement durable et la transition vers l'agroécologie. Ce travail, basé sur une étude de cas dans le nord de l'Argentine, analyse les relations tangibles et intangibles par lesquelles une communauté andine crée sa réalité et construit un paysage complexe et varié. L'étude emploie des méthodologies ethnographiques, combinant l'observation des participants avec des entretiens et une analyse qualitative. Le résultat met en évidence le réseau de liens entre humains et non-humains relatés par les personnes interrogées, tels que l'affection et la réciprocité, qui sont décisifs pour continuer à cultiver la terre et se développer d'une façon endogène - fondée sur une culture paysanne qui part des ancêtres. En outre, nous nous interrogeons sur la compatibilité de cette culture - dont l'origine est une immersion totale dans le paysage - avec les modes de vie actuels.

Mots-clés: agrobiodiversité; formes de connaissance; paysage culturel; relation hommenature; transformation

Abstract. The knowledge of ancestors: Key to the linkages with Mother Earth in an Andean community in Argentina

If we assume that landscapes are produced in permanent co-creation between humans and non-humans, it is relevant to recognize the forms of knowledge immersed in this process to design the socio-ecological transformation towards sustainable development, especially the transition to agroecology. This case study, which was carried out in northern Argentina, addresses the tangible and intangible relationships with which an Andean community builds its reality and a complex and varied landscape. The study uses ethnographic methodologies combining participant observation with interviews and qualitative analysis. The results reveal the network of links between humans and non-humans narrated by the people interviewed, such as affection and reciprocity, which are decisive to continue cultivating the land and to develop endogenously with their peasant knowledge based on their ancestors' knowledge. In turn, the compatibility of such knowledge is discussed - whose origin is a full immersion in the landscape - with current lifestyles.

Keywords: agroecology; forms of knowledge; human-nature relationship; rural landscapes; transformation 


\author{
Sumario \\ 1. Introducción 4. Resultados \\ 2. Consideraciones teóricas \\ 5. Discusión y conclusión \\ 3. Consideraciones metodológicas \\ Referencias bibliográficas
}

\title{
1. Introducción
}

Ciertos paisajes rurales complejos pueden resultar ser de un valor extraordinario para la humanidad. Algunos de ellos han sido destacados por su valor estético, histórico y cultural, como la Quebrada de Humahuaca en Argentina, nominada Paisaje Cultural ${ }^{1}$. Otros, por la resiliencia de sus ecosistemas de alta agrobiodiversidad, como la agricultura andina en Puno, Perú, elegida como uno de los sistemas importantes del patrimonio agrícola mundial (SIPAM $)^{2}$. Estos paisajes fueron generados a lo largo de los siglos por una gran diversidad de prácticas y estrategias de supervivencia de sus comunidades locales. Sin embargo, están siendo abandonados por causa de la emigración y la falta de viabilidad económica, además de amenazados por la desaparición de las formas de vida y de los conocimientos locales específicos que les dieron lugar.

Por otro lado, las áreas más productivas de la tierra están siendo sometidas a unas prácticas agrícolas crecientemente tecnificadas que permiten cultivar grandes superficies en poco tiempo y que, de este modo, van simplificando el sistema agroecológico. La agroindustria y ciertos servicios de asesoramiento sugieren paquetes tecnológicos para incrementar la producción, que no solo conducen a la pérdida de diversidad y de resiliencia de los ecosistemas, sino que también reducen las formas del saber a la adopción de dichas tecnologías. Estos sistemas, altamente competitivos económicamente, y conocidos como agricultura industrial, agravan el éxodo de la población rural, que en Argentina, entre 1947 (año de mayor población rural registrada) y 2010, disminuyó del 38 al 9 por ciento (Sili et al., 2015). Todo este proceso ha conducido a una progresiva desvinculación de grandes sectores de la sociedad respecto a la procedencia de sus alimentos.

Para contrarrestar esa tendencia de base extractiva, se está impulsando el concepto de agroecología desde diferentes organizaciones internacionales, gobiernos y movimientos sociales. Por ejemplo, la Organización de las Naciones Unidas para la Alimentación y la Agricultura (FAO) está definiendo el concepto de agroecología en relación con los objetivos de desarrollo sustentable (FAO, 2019), o bien el gobierno uruguayo ha incorporado la agroecología en

1. <http://whc.unesco.org/en/list/422> [Consulta: 21 de febrero de 2019].

2. <http://www.fao.org/giahs/giahsaroundtheworld/designated-sites/latin-americaand-the-caribbean/es/> [Consulta: 21 de octubre de 2019]. 
su legislación ${ }^{3}$. Uno de los principios de la agroecología es el intercambio de saberes y la reactivación de los conocimientos locales (Francis et al., 2017; Gliessman, 2017; Wezel et al., 2018; Anderson et al., 2019). Estudios recientes señalan, a su vez, que los procesos formativos agroecológicos tienen una componente territorial importante (McCune y Sánchez, 2019). Sin embargo, aún falta profundizar sobre la complejidad de los paisajes culturales o rurales agrobiodiversos que guardamos en la memoria, como los mencionados más arriba, y sobre el hecho de que dichos paisajes se generan con formas de conocimiento que nacen de la inmersión en los mismos, y que no solamente son controladas por el razonamiento abstracto, sino también desde el sentir (Ingold, 2011). Además, cabe no perder de vista que dichas formas de saber hoy no son hegemónicas, sino marginadas (Bidaseca et al., 2011).

Este trabajo se propone explorar, en un estudio de caso, la multiplicidad de dimensiones de conocimientos y afectos involucrados en la relación entre humanos y no humanos, y que, por sus cualidades, generan paisajes rurales agrobiodiversos. Para ello, primero analizaremos el marco institucional y económico del lugar, así como también el marco discursivo en el que tiene lugar la vida cotidiana (Berger y Luckmann, 1968; Wylie, 2007), para luego explorar las formas del saber con las cuales los entrevistados buscan restablecer su paisaje originario.

El caso de estudio es Nazareno, una comunidad andina remota en el norte de Argentina, provincia de Salta, que entiende estar conviviendo con la Madre Tierra. Se trata de un sitio de alto valor paisajístico, no solo por su belleza natural, inmersa en serranías de hasta 6.000 metros de altura, sino también por la circunstancia del mantenimiento del uso tradicional de la tierra y del cultivo en terrazas. Puede decirse que este paisaje, si bien más agreste, equivale al de la mencionada Quebrada de Humahuaca, ubicada al suroeste, del otro lado de la Sierra Santa Victoria, y que figura como Paisaje Cultural en la lista de la UNESCO desde el año 2003; entre otras razones, por su diversidad de variedades de maíz y sus múltiples usos culinarios (Cámara Hernández y Cabezas, 2007). Estos paisajes de alta montaña no son aptos ni para la intensificación ni para la mecanización agrícola, por la inaccesibilidad de las pendientes, la altura sobre el nivel del mar, las condiciones climáticas y los suelos someros (Ramos et al., 2013). Por este motivo se consideraron lugares apropiados para el estudio de la relación entre humanos y no humanos.

Los datos fueron relevados con métodos etnográficos, observación participante, discusiones grupales, entrevistas semiestructuradas y también narrativas recorriendo el paisaje (walking interviews), e interpretados luego mediante análisis cualitativo.

A continuación, el texto introduce el concepto de paisaje utilizado. Los capítulos siguientes profundizan en la metodología aplicada y en los resultados obtenidos. Finalmente se discute sobre la vinculación entre los saberes, los afectos y el paisaje producido por la comunidad estudiada.

3. Ley n. ${ }^{\circ} 19.717$, Plan Nacional para el fomento de la producción con bases agroecológicas, 23 de enero de 2019. 


\section{Consideraciones teóricas}

Desde la ecología del paisaje y la geografía se trabaja con el concepto de paisaje para focalizar la interrelación entre la sociedad y su entorno (Wylie, 2007; Termorshuizen y Opdam, 2009; Tengberg et al., 2012; Plieninger et al., 2014). Este trabajo entiende el término paisaje no solamente en el sentido de entornos extraordinarios de valor universal, como los mencionados más arriba, sino, en el sentido de la definición del Convenio Europeo del Paisaje (CEP), como: «cualquier parte del territorio tal como la percibe la población, cuyo carácter sea el resultado de la acción y la interacción de factores naturales y/o humanos» (Council of Europe, 2000, traducción). Aún más, entendemos al paisaje no en el sentido musealizado, sino performativo, lo que incluye las prácticas, los sentidos y los sentimientos experimentados, al cohabitar en él los humanos y no humanos (Cloke, 2016). Es decir, paisajes que no surgen (solamente) desde la visión en perspectiva del experto, sino desde la inmersión en el mismo (en el sentido del being-in-the world) (Ingold, 2002; Wylie, 2007; Steinhäuser, 2020).

Según Tim Ingold (2011), los humanos no transitan por un mundo hecho de antemano, sino que lo generan en permanente cocreación. Eso significa, continúa, reconocer una ecología del sentimiento (sentient ecology) que está asociada a determinadas formas del saber:

It is knowledge not of a formal, authorised kind, transmissible in contexts outside those of its practical application. On the contrary, it is based in feeling, consisting in the skills, sensitivities and orientations that have developed through long experience of conducting one's life in a particular environment. (Ingold, 2002: 25)

Entonces, los saberes no son una entidad desvinculada del paisaje, sino que, añade Wylie (2007), están inmersos en discursos, hábitos y formas de vida de una sociedad, lo que crea los paisajes típicos para un lugar y una sociedad en un momento dado. Bruno Latour postula que aceptar el Antropoceno como época actual en que el ser humano tiene la capacidad de actuar de manera inéditamente inconmensurable sobre los ecosistemas debería conducir a una profunda reflexión sobre la relación entre los humanos y la naturaleza que ha hecho posible tal uso desproporcionadamente extractivo de la Tierra (Latour, 2018). Y continúa afirmando que las ciencias naturales modernas están haciendo investigación, en su inmensa mayoría, de manera desvinculada, es decir, desde la perspectiva y suprimiendo la sensibilidad. Entender a la naturaleza como objeto separado del humano, según Descola (2014), es la base ontológica para su (sobre)explotación como recurso. Los autores coinciden en afirmar que la desvinculación y el desafecto entre humanos y no humanos es una explicación posible para el grave deterioro ecológico actual.

En este sentido, Ingold (2011: 75) profundiza en cómo, en la ciencia, la desvinculación del saber con el «objeto» de estudio hace imposible conocerlo:

But science as it stands rests upon an impossible foundation, for in order to turn the world into an object of concern, it has to place itself above and 
beyond the very world it claims to understand. The conditions that enable scientists to know, at least according to official protocols, are such as to make it impossible for scientists to be in the very world of which they seek knowledge. (Ingold, 2011: 75)

Según Latour, la transformación social hacia la sostenibilidad en las sociedades modernas podría conectarse con los conocimientos tradicionales locales; y esto no se trataría de la musealización de paisajes, sino de entender a las personas, nuevamente, en su arraigo a la tierra y cultivando vínculos entre los seres vivos (Latour, 2018).

Este trabajo interroga la percepción del paisaje de una comunidad rural andina a través de sus narraciones sobre su cosmovisión, basada en los saberes de sus ancestros. A su vez, observa qué estrategias desarrollan para recuperar y adaptar esos saberes a su situación actual y su proyección al futuro.

\section{Consideraciones metodológicas}

\subsection{Sitio de estudio}

La municipalidad de Nazareno está ubicada a 1.700 kilómetros de la capital de Argentina, Buenos Aires, y a 500 kilómetros (9 horas de viaje, en parte por camino sinuoso) de la capital provincial, Salta, donde se encuentran las autoridades centrales, los hospitales especializados, la educación terciaria, etc. Consta de 14 comunidades y asentamientos dispersos, y cuenta, en total, con alrededor de 3.000 habitantes ${ }^{4}$. La comunidad central, también llamada Nazareno, dispone de diferentes organismos públicos, municipalidad, hospital, policía, guarderías y escuelas de nivel primario, secundario y terciario. La Iglesia católica es predominante y tiene templos propios en las comunidades más pobladas; las iglesias evangélicas, mucho menos visibles, practican cultos en casas particulares. Las comunidades se inscribieron en el Registro Nacional Argentino de Pueblos Indígenas ${ }^{5}$ y crearon la Organización de Comunidades Aborígenes de Nazareno (OCAN), que desarrolla diferentes estrategias de protección de su cultura y su territorio andinos. Un ejemplo notorio fue no permitir la llegada de turismo insostenible, como ocurrió en la Quebrada de Humahuaca después de su nominación como Patrimonio de la Humanidad. Allí, la población originaria que con sus múltiples saberes había dado lugar al paisaje fue ampliamente marginada por la llegada masiva de viajeros, el aumento del valor de las tierras y la banalización de su cultura para fines comerciales (Bidaseca et al., 2011).

Estos paisajes tradicionalmente ofrecen una alta agrobiodiversidad por el cultivo en terrazas a diferentes niveles de altura (Hilgert y Gil, 2005) y por el pastoreo en trashumancia (Quiroga Mendiola, 2004). Los productos cosechados fueron y aún son utilizados a lo largo del año para elaborar diferentes

4. Datos del hospital de Nazareno, 2015.

5. Resolución SDS n. ${ }^{\circ}$ 4084, 30 de diciembre de 1998. 
platos estacionales, para alimentar al ganado o para ofrecer ritos a la Madre Tierra (Cámara Hernández y Cabezas, 2007; Ramos et al., 2013). Cultivar la tierra, manejar la cosecha y elaborar sus productos de esta manera supone el dominio de habilidades y saberes locales y específicos, basados en la observación y en la experiencia práctica. "Habría que pagarles por lo que saben hacer» (Julián Cámara Hernández, comunicación personal del 15 de marzo de 2016).

\subsection{Colección y análisis de datos}

Los estudios de campo se realizaron durante tres visitas en los años 2015 a 2017. Antes de empezar a trabajar con las comunidades fue necesario haber solicitado autorización al obispo de la prelatura de Humahuaca. Luego de las consultas correspondientes la visita fue anticipada por los altavoces del pueblo y por las radios locales. En el marco de la investigación se convivió en el pueblo y se pudo participar en quehaceres cotidianos, festividades, misas, clases del colegio secundario, rondas de discusión en la escuela terciaria y talleres de encuentro de la comunidad. Se realizaron, a personas de edades y sexos diferentes, 10 entrevistas semiestructuradas a expertos en su función como representantes de las distintas instituciones (Bogner et al., 2018) y 12 entrevistas narrativas recorriendo el lugar (walking interviews) (Jones et al., 2008; Carpiano, 2009; Evans y Jones, 2011), mediante contactos con estos participantes por muestreo en cadena; así como múltiples entrevistas etnográficas espontáneas combinadas con la observación participante (Flick, 2014).

Nuestra condición ajena al lugar condujo a múltiples explicaciones sobre la realidad local de la vida cotidiana (Berger y Luckmann, 1968), así como también a silencios, que, según Ephratt (2011), son parte de la comunicación y pueden constituir meros elementos para estructurar la conversación o bien ofrecer espacios de contenidos inexpresables (místicos, éticos, estéticos), o también ser omisiones estratégicas. En este contexto, la reflexión sobre el propio rol y trasfondo teórico, el proceso conjunto de generación de conocimientos, así como las visiones de la comunidad se fueron registrando en forma de notas y un diario de campo (véase Guber, 2011).

Las entrevistas, narraciones, observaciones y notas fueron integradas en un análisis cualitativo de codificación orientado en la teoría fundamentada para generar «datos ricos». Estos, según Charmaz (2014: 14), revelan las visiones, las intenciones, los sentimientos y las acciones de los participantes. Para profundizar la interpretación en este sentido, se dieron diferentes colores a pasajes de las transcripciones en base a determinados elementos verbales o prosódicos (Bänziger y Scherer, 2005; House, 2006; Richter, 2015), así como luego a los códigos correspondientes. Estos colores representan intenciones (lila), sentimientos (rosa) y acciones (verde) (véase la figura 2) de manera no excluyente. Las personas entrevistadas fueron anonimizadas de la manera siguiente: el tipo de entrevista (walking interview, WI; entrevista a expertos, EI) seguido por un número al azar, el sexo, la edad aproximada y el rol de la persona por el cual fue entrevistada. Por ejemplo: «WI4-f-40, campesina». 


\section{Resultados}

Se detallará, en primer lugar, el marco institucional donde está inmersa la comunidad y cuáles son sus opciones económicas. Luego se analizará el marco discursivo con el que se identificaron los entrevistados, que, según Wylie (2007), marca las pautas de las prácticas que una comunidad considera normales, por ejemplo: el tiempo que insume hacer uso de la tierra o elaborar platos para determinados festejos. Finalmente se explorará qué saberes creen necesarios para regenerar los paisajes rurales agrobiodiversos que en sus narraciones cultivaban sus ancestros.

\subsection{Marco institucional y económico}

La Iglesia católica tiene un papel central en la integración de las comunidades. La prelatura de Humahuaca fue fundada en 1970 después del Concilio Vaticano II. Ante la situación social observada (se trata aún hoy de una de las regiones más pobres de la Argentina) se compenetró con la cosmovisión indígena y la inculturó en su pastoral (Pliego, 2006; Olmedo, 2016) fundando, al mismo tiempo, la Obra Claretiana para el Desarrollo (OCLADE), para así ofrecer asistencia social (Occhipinti, 2013). La inculturación, según el obispo de la prelatura, da a sus conciudadanos andinos el sentido de pertenecer a una sociedad pluriétnica y de obtener una posición reconocida en la sociedad (Pliego, 2006; prólogo de Olmedo, 2016).

La Organización de Comunidades Aborígenes de Nazareno (OCAN) se propone integrar sus comunidades, realizar actividades culturales y gestionar el reclamo de propiedad comunitaria de las tierras. Forma parte de la identidad indígena ser pequeño agricultor de subsistencia. La OCLADE ha participado activamente en el proceso de fundación de OCAN. Durante las estadías con las comunidades pudo observarse que líderes de la OCAN cumplen roles centrales en las actividades de la iglesia. Lo cristiano y lo nativo se amalgama: «Nosotros decimos: Pachamama, Santa Tierra, que estemos bien» (WI4-f-40, campesina), pero otras iglesias cristianas minoritarias del pueblo no comparten este discurso.

Otras instituciones importantes son el hospital, que brinda asistencia programada aún a las comunidades más alejadas, así como las guarderías y las escuelas. Nazareno cuenta con un colegio secundario y un instituto de formación terciaria para maestros interculturales bilingües.

Todas las personas entrevistadas se identificaron como campesinas y campesinos actualmente o en el pasado inminente. Rara vez esta era su única actividad, sino que la combinaban, por ejemplo, con un comercio o una función pública. En el período de relevamiento de datos una fuente de ingresos eran los subsidios estatales, que no parecían causar el beneficio que debieran por falta de criterio en su concepción desde la ciudad. Cabe destacar que el turismo, que es fuente de ingresos en muchos sitios de la región, fue limitado solamente al turismo comunitario, excluyéndose el acceso a emprendimientos gestionados por personas ajenas a las comunidades locales. 
Figura 1. Vista sobre una comunidad flanqueada por los campos utilizados de acuerdo con los diferentes escalones ecológicos de montaña para la agricultura y la ganadería

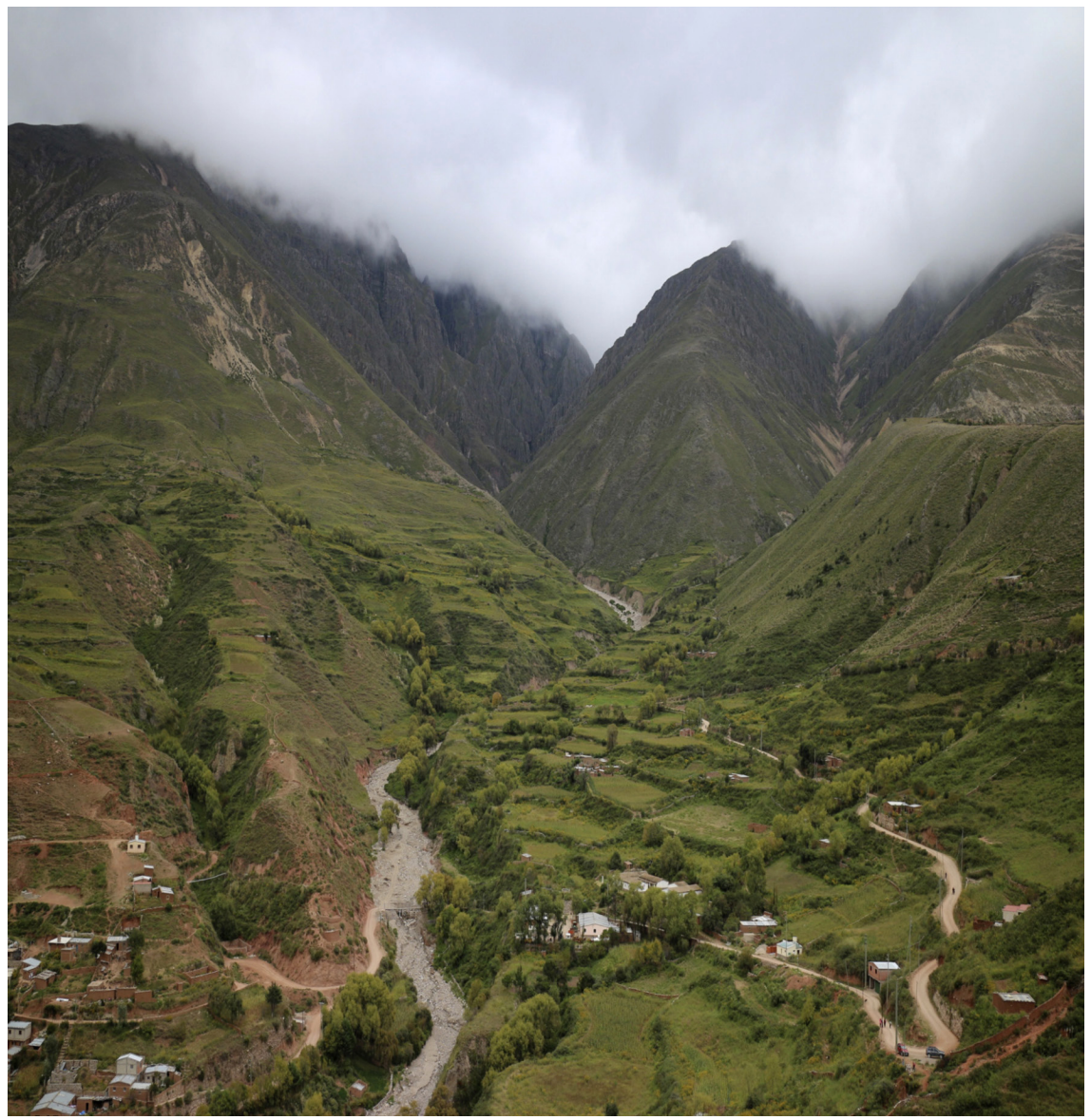

Fuente: Cornelia Steinhäuser.

Las actividades agropecuarias son mayoritariamente de subsistencia, y no cuentan, especialmente para las mujeres, como trabajo o actividad económica, sino como quehaceres domésticos (véase también Reboratti, 2009). Las prácticas agrícolas y de trashumancia, si bien atenuadas por la urbanización y la modernización de estilos de vida, estructuran las rutinas diarias y estacionales de la comunidad y dan lugar a una variedad de campos de cultivo, pastos, caminos, arboledas, pircas y asentamientos.

Se cultivan varios tipos de maíz, además de legumbres, papa, oca, quinoa, hierbas, flores y otros cultivos. No se utilizan maquinarias agrícolas ni agroquímicos. El agua de riego se maneja con acequias comunitarias. El suelo se 
fertiliza con deyecciones animales recogidas en el cerro. El ganado (caprino, ovino, bovino, camélidos) se cría con diferentes grados de trashumancia. No se llevan registros ni de volúmenes cosechados ni de parámetros ambientales.

Desde que se construyó la ruta de acceso en la década de 1990, una parte de la población que había emigrado retornó al pueblo, porque ya era posible optar por alternativas laborales o posibilidades de formación. La migración temporal por trabajo (cosecheros, etc.) tiene lugar todos los años. Algunos meses migran solamente los hombres, otros meses casi toda la familia. Otros emigran para estudiar, y frecuentemente no vuelven. Las personas entrevistadas, que son quienes permanecieron en las comunidades, percibían la ciudad mayoritariamente como un lugar peligroso, ruidoso y a veces denigrante.

\subsection{Pertenecer a una comunidad con identidad cultural}

Todos los participantes se adjudicaron una identidad cultural andina - refiriéndose a sí mismos como nativos, coyas, originarios o pertenecientes a la comunidad - en algún momento de la entrevista. La identidad cultural se describe como una cosmovisión que atribuyen a sus «ancestros» o «abuelos» — sin especificar cuántas generaciones hacia atrás- Esta cosmovisión se caracteriza por la reciprocidad entre las personas y la Madre Tierra; la agricultura de subsistencia, y una forma de vida austera, moderada y hospitalaria (figura 2). Esta identidad es reafirmada activamente por actores centrales de las comunidades, apuntando a la dignidad del trabajo de campo, así como al sentido comunitario, asociado, no por último, al reclamo de tenencia comunitaria de las tierras. Muchos entrevistados hablaban de sí en plural.

La reciprocidad se entiende como la conciencia del bienestar del otro. Es decir, no como una retribución igualitaria de valores o mercaderías, sino como la moderación en función de lo que cada uno puede ofrecer. Las familias campesinas que siguen en actividad sienten un vínculo de reciprocidad con la Madre Tierra (Pachamama) e integran este concepto en la comunidad (figura 2). La Madre Tierra ofrece espacio, alimento y, a su vez, impone reglas y tiempos. Para mantener una buena relación con ella se practican ritos cotidianos, como el agradecimiento de "chayar», que consiste en convidar a la Pachamama, o bien el apilamiento de piedras en las "apachetas» ubicadas en las abras para pedir protección para el camino.

La capacidad de practicar agricultura de subsistencia y saber cultivar la tierra de montaña es reconocida y supone una reafirmación de pertenencia al lugar (figura 2). Sin embargo, existe una conciencia creciente de que muchos saberes locales ya no están presentes. Paralelamente a las formas tradicionales, se han ido adoptando hábitos de uso del tiempo y de consumo de la sociedad moderna que resultan en una ruptura con la Madre Tierra. Algunos entrevistados dicen que les causa dolor ver campos sin cultivar. Esto no solo representa una pérdida económica, sino también una debilitación del carácter del paisaje (como lo define la CEP) en referencia a la identidad indígena local. Las preguntas son: ¿Cómo seguir manteniendo una forma de vida coherente con la 
Figura 2. Cosmovisión de la comunidad según los entrevistados. Vista en red de códigos y citas representativas (atlas.ti)

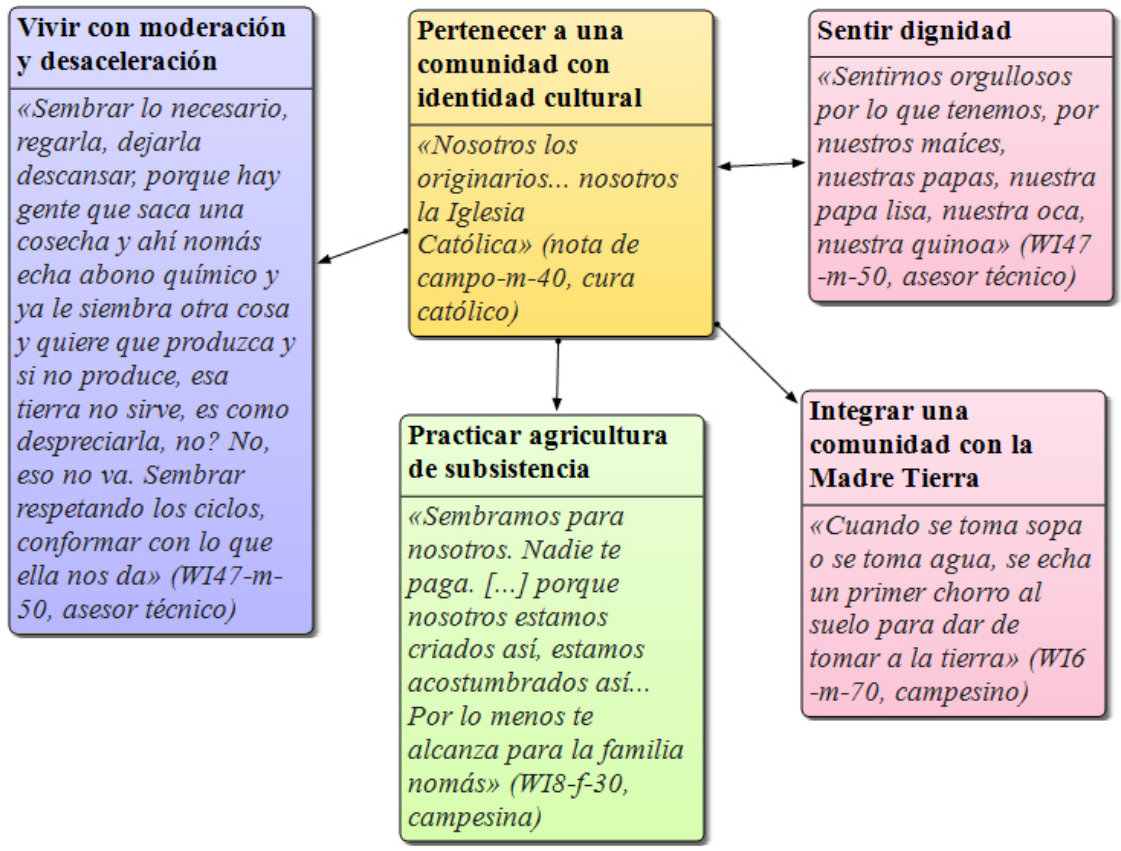

Fuente: elaboración propia.

identidad cultural andina y el uso correspondiente de la tierra? ¿Cómo subsistir sin turismo como opción económica?

\subsection{Desarrollar los saberes ancestrales como estrategia para permanecer}

En medio de esta tensión entre lo tradicional y lo moderno las diferentes instituciones y muchos pobladores llevan adelante iniciativas para poder permanecer en el lugar a pesar de las limitadas alternativas económicas. El presente trabajo se centra en las prioridades y en las tensiones observadas en las comunidades con respecto a las formas de desarrollar conocimientos, agrupadas en tres áreas temáticas: la selección de saberes debido a la ubicación alejada, la reflexión respecto al uso comunitario de los recursos comunes y la realimentación de saberes para reafirmar la identidad cultural (tabla 1).

Ante las necesidades de adaptación de estilos de vida, la ubicación geográfica remota de la comunidad es considerada, por algunos, como una oportunidad para promover un desarrollo autodeterminado, así como criterioso a la hora de incorporar saberes y cambios «no originarios» (tabla 1). Sin embargo, en cuanto a la selección de saberes, esta estará circunscrita a quienes tienen medios 
Tabla 1. Códigos y citas representativas indicando la situación geográfica y el sentimiento de pertenencia de las comunidades, así como las formas de conocimiento y las acciones correspondientes que desarrollan para permanecer en el lugar

\begin{tabular}{|c|c|}
\hline Código & Cita representativa \\
\hline Estrategias para permanecer & $\begin{array}{l}\text { «Lo que pasa es que faltan políticas públicas o comunitarias, } \\
\text { territoriales, que tienen que apuntar a lo que sabe hacer la } \\
\text { gente.» (WI50-m-40, político) }\end{array}$ \\
\hline Vivir en un lugar alejado & $\begin{array}{l}\text { "Por ahí uno se pone a pensar: "Por qué se habrán venido a vivir } \\
\text { aquí tan lejos?"» (WI52-f-30, comerciante) }\end{array}$ \\
\hline Incorporar saberes de afuera & «Avanzar a paso propio.» (El1-m-60, político) \\
\hline Incorporar cambios & $\begin{array}{l}\text { «Che, ¿cómo están las cosas, qué tenemos que hacer, qué } \\
\text { podemos hacer, qué no podemos hacer?» (WI47-m-50, asesor } \\
\text { técnico) }\end{array}$ \\
\hline Sentir pertenencia a un lugar & $\begin{array}{l}\text { «Nosotros sentimos que la tierra nos alimenta, nos da frutos, } \\
\text { y de la tierra vivimos.» (WI4-f-40, campesina) }\end{array}$ \\
\hline $\begin{array}{l}\text { Reflexionar sobre los bienes } \\
\text { comunes }\end{array}$ & $\begin{array}{l}\text { «Si no la cuidamos... en algún momento nos estamos suicidan- } \\
\text { do nosotros mismos.» (WI47-m-50, asesor técnico) }\end{array}$ \\
\hline Dar valor por la dedicación & «Hay que ponerse las pilas nomás.» (Wl8-f-30, campesina) \\
\hline Generar bienes comunes & $\begin{array}{l}\text { «[...] el próximo lunes todos a limpiar la acequia. No necesita- } \\
\text { mos que alguien nos convoque, eso ya viene por años.» (WI47- } \\
\text { m-50, asesor técnico) }\end{array}$ \\
\hline Formarse en el paisaje & $\begin{array}{l}\text { «Eso blanquito son ovejas. Al frente, derecho, ¿ve? Bueno ahí } \\
\text { chicos están paseando [las ovejas] y están cantando para no } \\
\text { aburrirse.» (WI } 42-f-30 \text {, estudiante terciario) }\end{array}$ \\
\hline $\begin{array}{l}\text { Integrar elementos del entorno } \\
\text { a la vida cotidiana }\end{array}$ & $\begin{array}{l}\text { «Uno lo ve tan obvio a cosas que ve cotidianamente. Y no lo } \\
\text { ves, digamos, porque vos siempre lo has tenido en la cabeza y } \\
\text { lo incorporás y no lo ves como algo que otra gente no lo sabe.» } \\
\text { (WI39-f-30, estudiante) }\end{array}$ \\
\hline Sentir pertenencia a un grupo & $\begin{array}{l}\text { «Saber bien cuál es nuestra raíz, cuál es nuestra costumbre, } \\
\text { de dónde venimos.»(Wl16-m-30, político) }\end{array}$ \\
\hline Trasmitir saberes & $\begin{array}{l}\text { «A mí me interesa que sepan, que sepan lo que aprendieron de } \\
\text { papá, lo que aprendieron de los abuelos.» (El54-m-40, profesor } \\
\text { de escuela) }\end{array}$ \\
\hline Compartir saberes & $\begin{array}{l}\text { «Hacer seguimiento con los productores y aprender también de } \\
\text { ellos, ¿no?» (WI47-m-50, asesor técnico) }\end{array}$ \\
\hline Recuperar saberes ancestrales & $\begin{array}{l}\text { «Los perdemos hace muchos años, y ahora, bueno, nosotros } \\
\text { buscamos volver a recuperarlos. No la tenemos tal como era } \\
\text { antes, bien viva, ¿estamos volviendo no?, y nos cuesta. Es } \\
\text { duro.» (WI37-m-50, OCAN) }\end{array}$ \\
\hline $\begin{array}{l}\text { Retroalimentar la cosmovisión } \\
\text { desde afuera }\end{array}$ & $\begin{array}{l}\text { «Se creó una escuela terciaria para la formación de maestros } \\
\text { dentro de la comunidad. Los estudiantes se dan nombres que- } \\
\text { chua y vienen docentes de afuera, muchos de la tecnicatura } \\
\text { indígena, a enseñar desde el idioma quechua hasta la confec- } \\
\text { ción de ollas de barro.» (Nota de campo) }\end{array}$ \\
\hline
\end{tabular}

Fuente: elaboración propia. 
y movilidad suficiente para formarse o buscar información fuera del lugar. Por otro lado, los medios de comunicación permiten acceder a todo tipo de información.

La pertenencia al lugar, el vínculo con la Madre Tierra y la dependencia de sus frutos para subsistir fue tema de reflexión en todas las entrevistas. Tal reflexión abarca desde la siembra hasta la cosecha, y desde el almacenamiento hasta la preparación de diferentes platos, de acuerdo con la estación del año. Cada uno de los pasos de elaboración requiere dedicación, creatividad y trabajo manual, y puede llevar días (tabla 1). Muchas actividades agrícolas tradicionalmente realizadas en comunidad, como el cuidado de las acequias, la atención del ganado o la siembra, siguen practicándose, si bien cada vez están más circunscritas al núcleo familiar. Con este proceso también se erosiona, imperceptiblemente, la capacidad de seguir evolucionando las normas sociales de acción colectiva en el manejo de los recursos, tal como las describe Ostrom (2000). Por otro lado, el formarse en el paisaje desde la niñez hace que se integren a la vida cotidiana elementos del entorno sin la conciencia de que en otros ámbitos lo normal sería comprarlos o reponerlos (tabla 1). Es así que el uso de los recursos silvestres para consumo (hierbas) o construcción (madera, tierra, grava) está poco regulado y se observan síntomas de sobreexplotación. Esto es particularmente notorio en los alrededores del pueblo, hacia donde migran los pobladores desde los sitios más alejados para tener acceso a los servicios. A esto se suman los residuos domiciliarios que tampoco formaban parte de la vida cotidiana de los ancestros y, por la ubicación remota del lugar, no son retirados hacia ninguna parte, sino que permanecen visibles. De este modo se genera una tensión entre aquellos saberes y reflexiones que hacen falta para el uso agrícola tradicional de subsistencia y la recolección de frutos y materiales, así como aquellos necesarios para estilos de vida actuales de consumo.

Se realizan múltiples actividades para reafirmar el sentido de pertenencia al grupo (inaugurar una antena para la radio local, procesiones y festividades de la Iglesia católica, la feria del trueque, etc.) (tabla 1). Sin embargo, la emigración, la descalificación de las formas de vida nativas en décadas anteriores o la urbanización, entre otros factores, están contribuyendo a promover la desagregación de las familias y las comunidades. Ante esta situación se busca recuperar la cosmovisión andina adquiriendo conocimientos teórico-prácticos en instituciones especializadas externas, las llamadas tecnicaturas. A su vez, se instauró una carrera terciaria bilingüe de formación docente en el pueblo mismo, para que dichos conocimientos fueran luego enseñados en las escuelas, por ejemplo: el idioma quechua, los saberes sobre cultivos o los trabajos artesanales. Para mantener la identidad cultural, los entrevistados mencionaron, muy frecuentemente, que era necesaria la transmisión de saberes, ya fuera en forma de diálogo de conocimientos entre campesinos, o bien la transmisión intergeneracional. Muchas veces aprenden los nietos de los abuelos, dado que los padres emigran para trabajar. Estos saberes adquiridos por experiencia y observación son altamente valorados, pero también escasos, puesto que, por ejemplo, durante el ciclo lectivo, niñas, niños y jóvenes pasan la mayoría del tiempo en las aulas. 


\section{Discusión y conclusión}

La revalorización de la cosmovisión andina, impulsada también por el movimiento indígena latinoamericano (Escobar, 2016), tiene una fuerte relevancia territorial y se está invocando en las negociaciones para los derechos de propiedad de las comunidades ${ }^{6}$. En este caso, la comunidad se atribuye una identidad étnica kolla, que, según observaciones de Yudi (2015: 239), logró «revertir la etnicidad como mote, como arcaísmo o como subalteridad [...] [convirtiéndose] en carisma grupal positivo». Escobar (2016: 118) considera que se trataría de «la redefinición de una supuesta identidad y realidad compartidas» de comunidades en busca de autonomía y de conexión con la Tierra y los seres vivos, que son vitales para su existencia. Esta conexión, continúa Escobar, no se encuentra únicamente en los conocimientos teóricos, sino que se expresa, especialmente, en las artes, los tejidos, las prácticas y las luchas de defensa de la Pachamama.

Los saberes vinculados a la cosmovisión de las comunidades estudiadas en este caso se circunscriben a las prácticas agrícolas. Si bien se están haciendo esfuerzos por reanimar el uso del telar y la producción alfarera, quedan pocos expertos locales y la viabilidad económica de tales actividades es marginal (en toda la región). Por otro lado, continúan practicándose ciertos ritos que contribuyen a fortalecer la identidad, así como el diálogo con la Madre Tierra para dar gracias y pedir protección. Sin embargo, en los colegios ocupa mucho más tiempo la educación formal, que muchos asumen que promete una mejor salida laboral. Esta transmisión de conocimientos, reconocen los entrevistados, no es equivalente a la que practicaban sus ancestros, que aprendían del entorno con la mente y el cuerpo. Según Tim Ingold (2011), para las cosmovisiones indígenas no hay un límite que separe el interior del exterior humano. Esto, argumenta, es esencial en las formas del saber:

Since the living body is primordially and irrevocably stitched into the fabric of the world, our perception of the world is no more, and no less, than the world's perception of itself - in and through us. This is just another way of saying that the inhabited world is sentient. (Ingold, 2011:12)

De lo contrario, es decir, ignorando los lazos vivos y afectivos entre el cuerpo y el entorno, se debilitan las capacidades íntimamente asociadas a un paisaje. Así también las prácticas comunitarias y los ritos a la Madre Tierra van perdiendo, imperceptiblemente, su fundamento.

Sin embargo, atribuir(se) una identidad indígena homogénea y predefinida, vivida localmente e imaginada desde la academia o el turismo, por un lado, y según argumenta Bryan (2012), podría excluir a quienes no pertenecen a la comunidad. Por el otro lado, tal como se observó en este caso de estudio, podría limitar el poder de adaptación a las dinámicas divergentes de opciones de vida

6. <https://www.argentina.gob.ar/derechoshumanos/inai/normativa>. [Consulta: 22 de enero de 2018] 
y de concepciones individuales de la Madre Tierra, así como a las tensiones que generan los cambios asociados a la globalización y a los medios de comunicación. En otras palabras, los cambios hacia los estilos de vida modernos no son compatibles con imaginarios de purezas étnicas, y ejercen impactos en el ecosistema que demandan flexibilidad para incorporar innovaciones, lo que implica incluir nuevas formas del saber.Las observaciones realizadas durante este estudio invitan a reflexionar sobre las sociedades actuales y a realizar la transformación socioecológica y la transición a la agroecología. Podemos constatar que las formas de vida observadas y narradas en la comunidad responden a expectativas globales de desarrollo sostenible asociadas al buen vivir y a una transformación de la sociedad hacia una mayor austeridad, eficiencia energética, moderación, creatividad, desaceleración y conciencia (Vanhulst y Beling, 2014). La capacidad de formar vínculos con la naturaleza para conformar un paisaje cultural o rural agrobiodiverso, estableciendo una relación íntima con un sitio y confiriéndole un carácter propio no es exclusiva de determinadas etnias que viven con la Madre Tierra, sino que se trata de una capacidad humana, como también argumentan Chan et al. (2016). En este sentido, según Fischer y Eastwood (2016), el humano puede agregar valor a un paisaje determinado con sus saberes - en nuestro caso la agrobiodiversidad de maíces en las terrazas andinas invirtiendo mucho tiempo y dedicación. Sin embargo, el frenesí de las formas modernas de vida conduce a paisajes rurales estandarizados, monótonos y acelerados. Los paisajes agrobiodiversos que generan una mayor resiliencia y dan origen a una diversidad genética más acusada, ya no parecen encajar en la relación actual entre economía y tiempo. Cabe relacionar esta circunstancia con las formas de conocimiento que convendría desarrollar. Latour (2018) argumenta que la transformación de la sociedad requiere la priorización de otras formas de saber; no solo las intelectuales, sino también las adquiridas a través de los sentidos.

De hecho, como reacción y alternativa a la agricultura industrial, está teniendo lugar una nueva territorialización de la agricultura que está asociada con sentimientos de pertenencia e identidad y que, según Ramírez-García et al. (2016), está conduciendo a cierto cambio en los paisajes rurales. En este sentido cabe seguir investigando sobre los vínculos tangibles e intangibles que se encuentran en estos nuevos paisajes y, especialmente, sobre los procesos de vinculación que tienen lugar en la generación joven, criada en las últimas décadas, que apenas conoce las formas del saber asociadas a los paisajes más complejos y que ha ido experimentando el desarraigo del sistema agroalimentario.

\section{Referencias bibliográficas}

Anderson, Colin R.; Maughan, Chris y Pimbert, Michel P. (2019). «Transformative agroecology learning in Europe: Building consciousness, skills and collective capacity for food sovereignty». Agriculture and Human Values, 36 (3), 531-547. $<$ http://dx.doi.org/10.1007/s10460-018-9894-0>

BäNZiger, Tanja y SCHERER, Klaus R. (2005). «The role of intonation in emotional expressions». Speech Communication, 46 (3-4), 252-267. $<$ http://dx.doi.org/10.1016/j.specom.2005.02.016> 
Berger, Peter L. y Luckmann, Thomas (1968). La construcción social de la realidad. Buenos Aires: Amorrortu, 1979.

Bidaseca, Karina; Borghini, Natalia; Salleras, Lucila y Millán, Facundo (2011). «Donde llega el diablo: Identidades exotizadas y procesos de patrimonialización en la Quebrada de Humahuaca». En: Borghini, Natalia y Bidaseca, Karina (coord.). Signos de la identidad indigena: Emergencias identitarias en el limite del tiempo histórico. Buenos Aires: SB, 47-74.

Bogner, Alexander; LitTig, Beate y Menz, Wolfgang (2018). «Generating Qualitative Data with Experts and Elites». En: FLICK, Uwe (coord.). The SAGE Handbook of Qualitative Data Collection. Londres: Sage, 652-665.

Bryan, Joe (2012). «Rethinking Territory: Social Justice and Neoliberalism in Latin America's Territorial Turn». Geography Compass, 6, 215-226. <http://dx.doi.org/10.1111/j.1749-8198.2012.00480.x>

Cámara Hernández, Julián y Cabezas, Dora A. (2007). Maíces andinos y sus usos en la Quebrada de Humahuaca y regiones vecinas. Buenos Aires: Editorial Facultad de Agronomía. Universidad de Buenos Aires.

CARPIAno, Richard M. (2009). "Come take a walk with me: the "go-along" interview as a novel method for studying the implications of place for health and well-being». Health of Place, 15 (1), 263-272. <http://dx.doi.org/10.1016/j.healthplace.2008.05.003>

Chan, Kai M.A.; Balvanera, Patricia et al. (2016). "Opinion: Why protect nature? Rethinking values and the environment». Proceedings of the National Academy of Sciences of the United States of America, 113 (6), 1462-1465. <http://dx.doi.org/10.1073/pnas.1525002113>

Charmaz, Kathy (2014). Constructing grounded theory: A practical guide through qualitative analysis. Los Angeles: Sage.

Cloke, Paul (2016). «Rural Landscapes». En: Johnson, Nuala C.; Schein, Richard H. y Winders, Jamie (coord.). The Wiley-Blackwell Companion to Cultural Geography. Chichester: Wiley-Blackwell, 225-237.

Council of Europe (2000). European Landscape Convention: ELC. Florencia.

Descola, Philippe (2014). Beyond nature and culture. Chicago; Londres: The University of Chicago Press.

Ephratт, Michal (2011). "Linguistic, paralinguistic and extralinguistic speech and silence». Journal of Pragmatics, 43 (9), 2286-2307. <http://dx.doi.org/10.1016/j.pragma.2011.03.006>

Escobar, Arturo (2016). «Desde abajo, por la izquierda, y con la Tierra: La diferencia de Abya Yala/Afro/Latino-América». Intervenciones en Estudios Culturales, 3, $117-134$.

Evans, James y Jones, Phil (2011). "The walking interview: Methodology, mobility and place». Applied Geography, 31 (2), 849-858. <http://dx.doi.org/10.1016/j.apgeog.2010.09.005>

FAO (2019). Scaling up agroecology to achieve the sustainable development goals: Proceedings of the second FAO international symposium. Roma: FAO.

Fischer, Anke y Eastwood, Antonia (2016). "Coproduction of ecosystem services as human-nature interactions-An analytical framework». Land Use Policy, 52, 41-50.

FLICK, Uwe (2014). An introduction to qualitative research. Londres; Thousand Oaks, CA; Dehli: Sage.

Francis, Charles; Breland, Tor et al. (2017). «Learning Agroecological Practices as Components of Complex Farming Systems». En: Wezel, Alexander (coord.). 
Agroecological practices for sustainable agriculture: Principles, applications, and making the transition. Singapur: World Scientific Publishing Company, 445-461.

Gliessman, Steve (2017). «The co-creation of agroecological knowledge». Agroecology and Sustainable Food Systems, 42 (1), 1. <http://dx.doi.org/10.1080/21683565.2017.1289727>

Guber, Rosana (2011). La etnografía: Método, campo y reflexividad. Buenos Aires: Siglo XXI.

HilgerT, Norma I. y Gil, Guillermo E. (2005). «Traditional Andean agriculture and changing processes in the Zenta River Basin, Salta, Northwestern Argentina». Darwiniana, 43 (1-4), 30-43.

House, Jill (2006). "Constructing a context with intonation». Journal of Pragmatics, 38 (10), 1542-1558. <http://dx.doi.org/10.1016/j.pragma.2005.07.005>

INGOLD, Tim (2002). The perception of the environment: Essays on livelihood, dwelling and skill. Londres; Nueva York: Routledge.

- (2011). Being alive: Essays on movement, knowledge and description. Londres; Nueva York: Routledge.

Jones, P.; Bunce, G. et al. (2008). «Exploring space and place with walking interviews». Journal of Research Practice, 4 (2), Article D2.

Latour, Bruno (2018). Down to Earth: Politics in the New Climatic Regime. Newark: Polity Press.

McCunE, Nils y SÁNCHEZ, Marlen (2019). «Teaching the territory: Agroecological pedagogy and popular movements». Agriculture and Human Values, 36 (3), 595-610. <http://dx.doi.org/10.1007/s10460-018-9853-9>

OcchipINTI, Laurie (2013). «Liberating Development: Religious Transformations of Development Discourse». Perspectives on Global Development and Technology, 12 (3), 427-443.

Olmedo, Jesús (2016). Una misión - Una iglesia: 47 años de la prelatura de Humahuaca. Humahuaca: Prelatura de Humahuaca.

Ostrom, Elinor (2000). «Collective action and the evolution of social norms». Journal of Economic Perspectives, 14 (3), 137-158.

Pliego, Manolo (2006). Sabiduría y espiritualidad indigena. Humahuaca: Prelatura de Humahuaca.

Plieninger, Tobias; Van der Horst, Dan; Schleyer, Christian y Bieling, Claudia (2014). «Sustaining ecosystem services in cultural landscapes». Ecology and Society, 19 (2).

Quiroga Mendiola, Mariana (2004). «Highland Grassland Vegetation in the Northwestern Andes of Argentina». Mountain Research and Development, 24 (3), 243-250. <http://dx.doi.org/10.1659/0276-4741(2004)024[0243:HGVITN]2.0.CO;2>

Ramírez-García, Susana; Mancha-Cáceres, Olga I. y Del-Canto-Fresno, Consuelo (2016). «Las agriculturas territorializadas: Oportunidades y retos frente al paradigma agroindustrial». Documents d'Anàlisi Geogràfica, 62 (3), 639. <http://dx.doi.org/10.5565/rev/dag.374>

Ramos, Rita S.; Hilgert, Norma I. y Lambaré, Daniela A. (2013). «Agricultura tradicional y riqueza de maíces (Zea mays): Estudio de caso en Caspalá, provincia de Jujuy, Argentina». Boletín de la Sociedad Argentina de Botánica, 48 (3-4), 607-621. <https://doi.org/10.31055/1851.2372.v48.n3-4.7623>

Reboratti, Carlos E. (2009). El Alto Bermejo: Realidades y conflictos. Buenos Aires: La Colmena. 
Richter, M. (2015). «Can you feel the difference?: Emotions as an analytical lens». Geographica Helvetica, 70 (2), 141-148. <http://dx.doi.org/10.5194/gh-70-141-2015>

Sili, Marcelo; Guibert, Martine y Bustos Cara, Roberto (2015). Atlas de la Argentina rural. Buenos Aires, Argentina: Capital Intelectual.

STEINHÄUSER, Cornelia (2020). «Mountain farmers' intangible values foster agroecological landscapes: Case studies from Sierra Santa Victoria in northwest Argentina and the Ladin Dolomites, northern Italy». Agroecology and Sustainable Food Systems, 33 (3), 1-26.

<http://dx.doi.org/10.1080/21683565.2019.1624285>

Tengberg, Anna; Fredholm, Susanne et al. (2012). "Cultural ecosystem services provided by landscapes: Assessment of heritage values and identity». Ecosystem Services, 2 (0), 14-26.

Termorshuizen, Jolande W. y Opdam, Paul (2009). «Landscape services as a bridge between landscape ecology and sustainable development». Landscape Ecology, 24 (8), 1037-1052.

Vanhulst, Julien y Beling, Adrian E. (2014). «Buen vivir: Emergent discourse within or beyond sustainable development?». Ecological Economics, 101, 54-63.

Wezel, Alexander; Goette, Julia et al. (2018). "Agroecology in Europe: Research, Education, Collective Action Networks, and Alternative Food Systems». Sustainability, 10 (4), 1214. <http://dx.doi.org/10.3390/su10041214>

WyLIE, John W. (2007). Landscape. Londres; Nueva York: Routledge.

Yudi, Raúl J. (2015). Kollas de Nuevo: Etnicidades, trabajo y clasificaciones sociales en los Andes de la Argentina. San Salvador de Jujuy: Purmamarka Ediciones. 